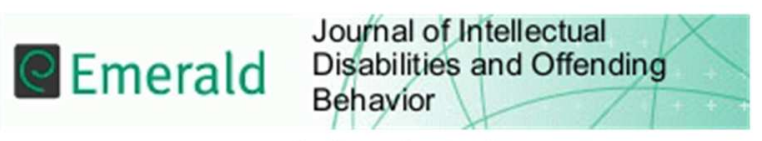

\title{
The Characteristics and Treatment Needs of Fire Setters with Intellectual Disability: Descriptive Data and Comparisons between Offence Type
}

\begin{tabular}{|r|l|}
\hline Journal: & Journal of Intellectual Disabilities and Offending Behavior \\
\hline Manuscript ID & JIDOB-10-2016-0019.R1 \\
\hline Manuscript Type: & Research Paper \\
\hline Keywords: & intellectual disability, fire setting, treatment, characteristics, needs, arson \\
\hline \multicolumn{2}{|c}{} \\
\hline
\end{tabular}




\title{
The Characteristics and Treatment Needs of Fire Setters with Intellectual \\ Disability: Descriptive Data and Comparisons between Offence Type
}

\begin{abstract}
Purpose: Fire setters who have an intellectual disability (ID) are often identified as posing a particular danger to the community although relatively little is known about their characteristics, treatment, and support needs.
\end{abstract}

Methodology: This study describes the characteristics of 134 residents of low, medium, and high security ID facilities in the United Kingdom who have either an index offence of arson, emparing them with these who have-a violentn index offence, involving either violent or a sexual indexat offenceding.

Findings: Index arson offenders who had an ID had multiple prior convictions, a history of violent offending, and a high likelihood of having a comorbid mental disorder. There were many shared characteristics across the three groups.

Practical implications: The current study suggests that offenders who have ID who set fires have treatment needs that are similar to those of violent and sex offenders. It follows that fire setters who have an ID may also benefit from participating in more established offending behaviour treatment programs, such as cognitive behaviour therapy programs, developed for other types of offender.

Originality: This study is one of the few which has investigated the characteristics and treatment needs of persons who have an ID who set fires. In particular, it is one of the first to compare the characteristics and treatment needs for persons with ID who set fires, to those who have committed violent and sexual offences. 
Keywords: intellectual disability; fire setting; treatment; characteristics; needs; arson

It has not been uncommon for $\underline{F}$ fire setters have often to-been identified as having an Intellectual Disability (ID) (Johnston, 2004; Inciardi, 1970; Fineman, 1995), and-yet there have been few empirical investigations into the characteristics of this group, and progress in the development of specialised interventions has been much slower than for offenders who have committed violent or sexual offences (Curtis, McVilly and Day, 2012). In fact, much of our current knowledge about fire setters with ID comes mostly from descriptive studies that were conducted 20 years ago. For example, Murphy and Clare (1996) described the characteristics of ten fire setters with ID whose ages ranged from 18 to $38(M=26.4, S D=7.5)$, with IQs, as measured by the Wechsler Adult Intelligence Scale-Revised (Wechsler, 1981), ranging from 60 to 78 $(M=68.4, S D=5.7)$. The fire setters with ID in this sample were reported to commonly feel angry, not listened to, and sad or depressed before setting fires. Harris and Rice (1996), in their study of 243 fire setters from a maximum security psychiatric institution, reported that of all the different groups described within their study, the 'multi fire-setter' group were the least intelligent, had a history of setting fires, and displayed high levels of aggression. This latter finding, relating to a previous history of aggression is noteworthy in light of evidence from some studies that fire setters with ID have low levels of aggression (Blanco, Alegria, Petry et al., 2010) with others reporting high levels and histories of violent offending (Dickens, Sugarman, Ahmad et al., 2007).

There have been numerous assertions that fire setters who have an ID are likely to repeat fire setting behaviour and engage in other offending (Devapriam, Raju, Singh et al., 2007). This has been attributed to limited social skills, impulsivity, 
and the inability to resist impulses to set fires (Johnston, 2004). Leong and Silva (1999) classified 32 fire setters into two groups, one of which was labelled 'cognitive pathology' and included persons with 'limited intellect' who were older and had a lengthy history of both criminal behaviour and fire setting. Labree, Nijman, van Marle, and Rassin (2010), however, suggested that although fire setters with ID do typically have lengthy criminal histories, the number of previous convictions they receive does not necessarily differentiate them from other offender groups, nor have these differed from fire setters without an ID (Dickens, Sugarman, Ahmad et al., 2008). It is also difficult to draw robust conclusions given that these studies described persons with 'low intelligence' or 'limited intellect', leaving it unclear whether participants had been formally diagnosed with an ID.

Mental disorders have been argued to be common for fire setters who have an ID. For example, Lees-Warley and Rose (2015) identified that psychiatric diagnoses were present in two thirds of the studies included in their systematic review regarding fire setters with ID. The identified disorders included Pervasive Developmental Disorder, Schizophrenia, Recurrent Depressive Disorder, Affective Disorder, Psychosis, Schizoaffective Disorder, Psychotic Depression, Alcohol Dependency, and 'Psychiatric Disorder'. Many studies have identified a diagnosis of Schizophrenia in fire setters, including those who have an ID (Anwar, Langstrom, Grann et al., 2011; Dickens, Sugarman, Ahmad et al., 2007; Enayati, Grann, Lubbe et al., 2008; Harris and Rice, 1996; Koson and Dvoskin, 1982; Repo and Virkkunen, 1997; Rice and Harris, 1991; Ritchie and Huff, 1999; Long, Banyard, Fulton et al., 2014; Murphy and Clare, 1996). Personality Disorder has also been commonly identified for fire setters who have an ID (Alexander, Chester, Green et al., 2015; Lees-Warley and Rose, 2015; Devapriam, Raju, Singh et al., 2007). It has been suggested, however, that psychiatric diagnoses may simply occur commonly among persons who commit 
crimes, or who engage in problematic behaviours, rather than playing any causal role (Anwar, Langstrom, Grann et al., 2011; Ducat, McEwan and Ogloff, 2013).

In summary then, fire setters who have an ID are a poorly understood population. Nonetheless, Taylor, Thorne, Robertson, and Avery (2002) have argued that they present a set of complex needs that must be addressed in treatment, and yet very few studies have been able to identify specific treatment needs. This includes the extent to which the offending reflects a broader pattern of anti-sociality. The aim of this study then is to examine the distinctive characteristics of arson offenders with ID who had been treated by the UK's National Health forensic mental health service. It compares those persons with ID who had an index offence of arson, a sexual index offence, or a violent index offence. The variables of investigation chesen for this study largely based on these used in previous stulies of persens with ID whe were known to have engaged in generaloffending behaviour (Hogue, Steptoe, Taylor

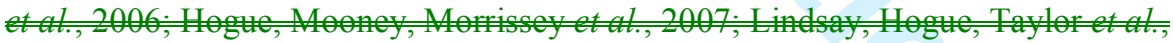
2008; O'Brien, Taylor, Lindsay et al., 2010), as well as on data that were available in the datase utilised in the current study.

\section{Method}

\section{Ethics Approval}

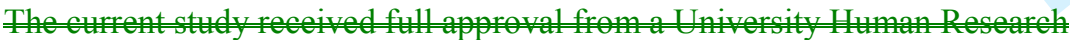

Ethies-Committee.

\section{Study Sites}

Three study sites contributed to the database, and were identified as L1, L2, and L3. All three sites were UK forensic mental health services, which accepted people who had a full-scale IQ of less than 75 and who presented with significant deficits in adaptive behaviour which were evident from childhood/adolescence 
(Hogue, Steptoe, Taylor et al., 2006). L1 is a high security site and is the national centre for high-secure ID services. L2 is a medium and low security site which provides inpatient forensic services for people with ID on a local, regional, and national basis. L3 is a community site, and comprises a 10-bed open unit and a large number of day places. These settings are described in detail by Hogue, Steptoe,

Taylor et al. (2006).

\section{Procedure and Materials}

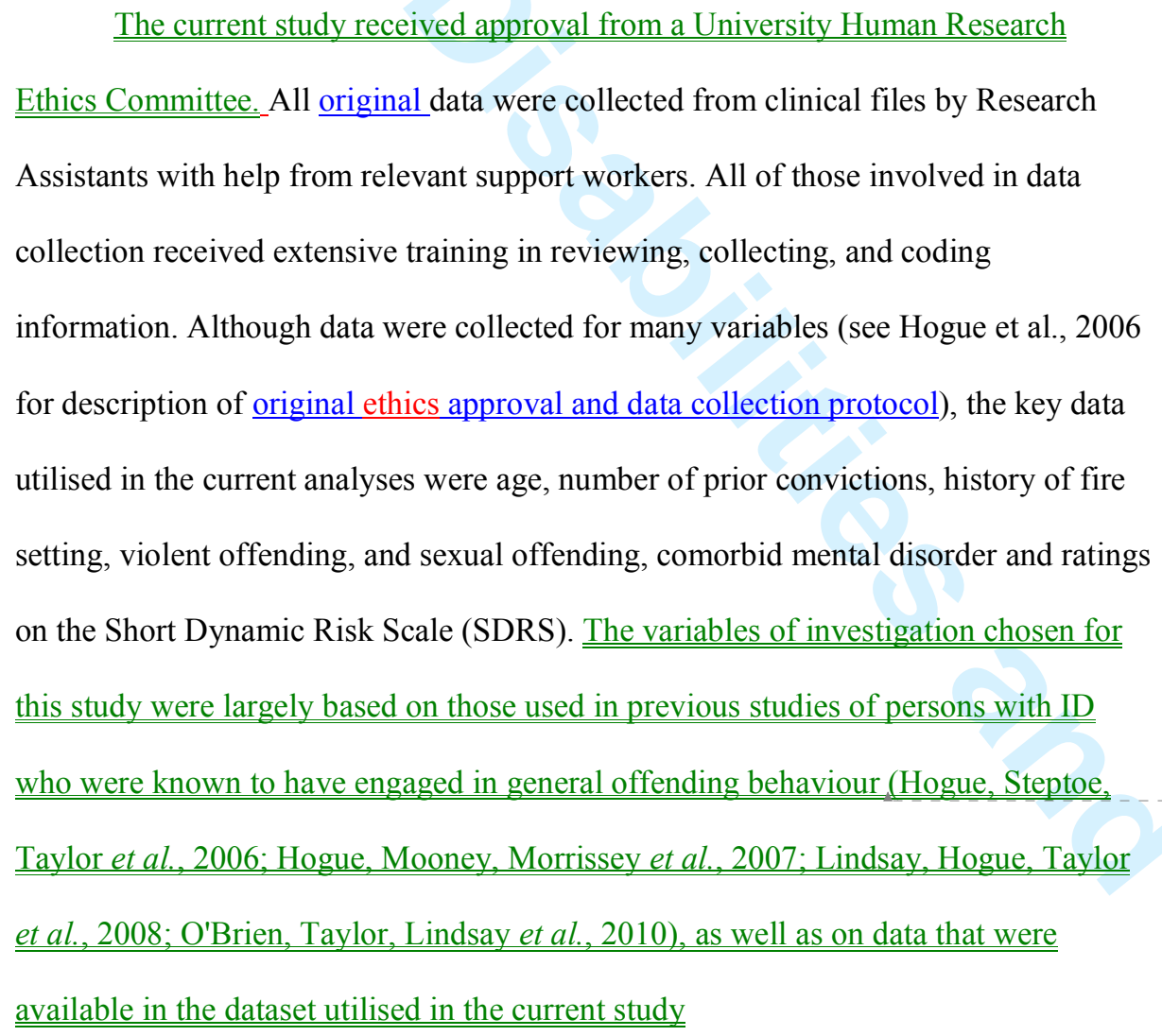

The SDRS is an eight-item scale that assesses a range of different dynamic risk factors/needs, such as hostile attitude, coping skills, self-care skills, and consideration of others. Ratings are made on a 0 to 4 scale (where $0=$ No problem, and $4=$ Severe problem). The scale has been shown to predict violent and sexual 
incidents with a high level of accuracy (Quinsey, Book and Skilling, 2004) however, no psychometric properties have been reported by the authors. In the current study the internal reliability of the scale, as measured by Cronbach's alpha, was 0.91 .

Offence information within the existing database was classified according to the original case notes, such that any violent offence was labelled in case notes as 'violent', any sexual offence was labelled as a 'sex offence', and any fire setting related offence was labelled as 'arson'. A separate variable indicated their index offence. It should be acknowledged that although an offender was classified in one group based on their index offence, he or she may still have had other convictions, or a reported history of other offending behaviour. Further, whilst the original database contained 212 male offenders with ID, the current study utilised only 134 of these offenders, specifically those who had violent, sexual, or arson-related index offences. As none of the variables to be used in the analyses met the assumption of normality, even after transformations were applied, non-parametric tests were used when appropriate.

ResultsAs none of the used in the analyses met the assumption of normality, even after transformations were applied, non parametrie tests were used when appropriate.

\section{Descriptive Statistics}

Data from a total of 134 male offenders with ID were used in the analysis. All had a diagnosis of Intellectual Disability. Of these, 114 were diagnosed using the International Classification of Diseases (ICD) criteria (World Health Organisation, $2010)$ as having a mild ID (85.7\%), 14 as having a moderate ID (10.5\%), one as having a severe ID, and five as having an unspecified ID (3.7\%).

As shown in Table 1, those with an offence of arson $(n=18)$ were, on average, the youngest at the time of admission to services, but they were on average the oldest 
group at the time of data collection (i.e., assessment). However, these differences were not significant between groups.

$<$ INSERT TABLE 1 ABOUT HERE $>$

\begin{abstract}
A Kruskal-Wallis Independent Samples $H$-test was used to compare the mean number of convictions prior to $18, \chi^{2}(2, n=134)=2.15, p=.34$, and mean convictions after $18, \chi^{2}(2, n=133)=3.06, p=.22$. No significant differences were found between index arson, index violent, or index sexual offenders. As might be expected, a history of fire setting was most commonly recorded in those participants who had an index offence of arson $\left(44.4 \% ; \chi^{2}(2, n=134)=15.71, p<.001, V=.34\right)$. A history of violent offending was most common for those with an index violent offence $\left(60.4 \% ; \chi^{2}(2\right.$, $n=134)=12.58, p<.01, V=.31$ ), and a history of sexual offending was most common for those with an index sexual offence $\left(52.4 \% ; \chi^{2}(2, \mathrm{n}=134)=13.91, p<.001, V=.32\right.$.) All groups were rated as experiencing high levels of comorbid mental disorder, with the highest rate experienced by the index violent offence group $\left(73.6 \% ; \chi^{2}(2\right.$, $n=133)=9.50, p<.01 ., \quad V=.27)$.
\end{abstract}

$<$ INSERT TABLE 2 ABOUT HERE $>$

A Kruskal Wallis $H$ Test was then used to determine differences between the three groups on each of the offender needs as measured by the SDRS. As shown in Table 3, there were no statistically significant differences between groups for any of the eight items.

$<$ INSERT TABLE 3 ABOUT HERE $>$

\title{
Discussion
}


There is a paucity of literature reporting on characteristics and rehabilitation needs of fire setters with ID. As such the aim of the current study was to investigate and compare the characteristics and needs of 134 fire settersoffenders with ID who are located in community, low, medium, and high secure sites in the $\mathrm{UK}_{2}$ who have an index offence of arson, a violent index offence, or a sexual index offence.

Although the index violent offenders had a relatively higher number of prior convictions than index arson or sexual offenders, the differences between groups were not statistically significant. This suggests that prior convictions and an extensive criminal history for other offences are relatively common for fire setters with ID. It follows that there is a need for treatment programs for this group to include treatment targeting a range of criminal behaviours, rather than only specialised treatment focussed on fire setting. Over one third of those with an index offence of arson also had a history of violent offences, despite previous studies reporting relatively low levels of violence in this group (Rice and Harris, 1991).

Three quarters of index violent offenders had been diagnosed with a comorbid mental disorder, compared with two thirds of those with an index arson offence. Those with an index sex offence were least often diagnosed with a comorbid mental disorder, with under half reporting a comorbid diagnosis. Rates of personality disorder were comparable between index violent offenders and index arson offenders, but far higher than for index sex offenders. For example, a diagnosis of Schizophrenia was far more common in those with an index violent offence. However, it is important to note that many fire setters who provided data used in this study were from a population of ID offenders who had been referred to secure mental health services. As such, they are more likely to have comorbid mental disorders, together with challenging and complex behaviours. 
In summary, the characteristics of index arson offenders with ID in this sample are: a history of fire setting behaviour; multiple prior convictions; a history of violent offending; and a high likelihood of having a comorbid mental disorder. These characteristics are shared by other offender groups, rather than being exclusive to fire setters. There is little to suggest that those with an index offence of arson present with treatment needs distinct from either index violent or index sex offenders with ID. Whilst this highlights the potential for arson offenders to attend similar treatment programs to other types of offender (i.e. violent or sexual offenders), this must be considered in the context of existing programs for offenders with ID. For example, a commonly used treatment program for sex offenders with ID is the Sex Offender Treatment Services Collaborative - Intellectual Disability (SOTSEC-ID) cognitive behavioural treatment, which includes components focussed on sex education and $\underline{\text { sexual offending behaviour in particular. Whilst these are important treatment targets }}$ for sex offenders with ID (Lindsay, 2002), they might not be relevant to all fire setters. Existing programs for violent offenders, that generally adopt a cognitive behavioural approach (Taylor, Novaco, Gilmer et al., 2002), on the other hand, may be more relevant and useful given the concepts addressed in these programs include those identified as relevant to fire setters through the current study, such as anger management. Whilst cognitive behavioural programs have been designed for fire setters (Curtis, McVilly and Day, 2012; Hall, Clayton and Johnson, 2005), these have not undergone extensive evaluation, and it is not clear whether they have benefits above and beyond other cognitive behavioural programs, such as those designed for violent behaviour, or behaviour change more generally. If such programs were able to $\underline{\text { reduce offending behaviour, including fire setting, for those with an ID, as might be }}$ expected given the lack of distinct characteristics and treatment needs identified in the 
current study, then this would have significant resource saving implications for treatment services.

Of course this study has some limitations. First, the majority of the sample were detained in secure settings, resulting in limited scope to generalise the findings outside of this context. Further, the majority fell into the mild ID range, which means that the findings might only apply to those with an index offence of arson who have a mild ID, rather than representing a broader range of persons with ID who set fires. A second limitation is that these data were collected for a prior study, and consequently only certain needs and characteristics were able to be investigated. In addition, data were collected solely from the original client case notes and as such were dependent on the quality of these (Hogue, Steptoe, Taylor et al., 2006), as well as the ability of the research assistants to collect these data accurately and consistently. There was also a relatively small proportion of persons with an index offence of arson within the sample, and no control group of offenders who would fit into the categories but who did not have an ID. A comparison with this latter group would have allowed direct distinctions to be drawn between fire setters who have an ID and those who do not.

Nonetheless, the findings of this study provide further evidence of the complexity of the rehabilitation and support needs of offenders with ID who set fires. Given they share many of the characteristics of violent and sex offenders, it is possible that they might benefit from participation in aspects of programs that have been developed for these groups. However, given what we know about the inherent problems this population experience with generalisation of learning, further work is needed with respect to the targeting of fire setting behaviours within existing programs. 


\title{
References
}

\author{
Alexander, R.T., Chester, V., Green, F.N., Gunaratna, I. and Hoare, S. (2015). Arson \\ or fire setting in offenders with intellectual disability: Clinical characteristics, forensic \\ histories, and treatment outcomes. Journal of Intellectual \& Developmental \\ Disability, Vol. 40 No. 2, pp. 189-197. \\ Anwar, S., Langstrom, N., Grann, M. and Fazel, S. (2011). Is arson the crime most \\ strongly associated with psychosis? A national case-control study of arson risk in \\ schizophrenia and other psychoses. Schizophrenia Bulletin, Vol. 37 No. 3, pp. 580- \\ 586. \\ Blanco, C., Alegria, A.A., Petry, N.M., Grant, J.E., SImpson, H.B., Liu, S.M., Grant, \\ B.F. and Hasin, D.S. (2010). Prevalence and correlates of fire-setting in the United \\ States: results from the National Epidemiologic Survey on Alcohol and Related \\ Conditions (NESARC). Journal of Clinical Psychiatry, Vol. 71, pp. 1218-1225.
}


Curtis, A., McVilly, K. and Day, A. (2012). Arson treatment programmes for offenders with disability: a systematic review of the literature. Journal of Learning Disabilities and Offending Behaviour, Vol. 3 No. 4, pp. 186-205.

Devapriam, J., Raju, L., Singh, N., Collacott, R. and Bhaumik, S. (2007). Arson: characteristics and predisposing factors in offenders with intellectual disabilities. The British Journal of Forensic Practice, Vol. 9 No. 4, pp. 23-27.

Dickens, G., Sugarman, P., Ahmad, F., Edgar, S., Hofberg, K. and Tewari, S. (2007).

Gender differences amongst adult arsonists at psychiatric assessment. Medicine,

Science and the Law, Vol. 47, pp. 233-238.

Dickens, G., Sugarman, P., Ahmad, F., Edgar, S., Hofberg, K. and Tewari, S. (2008).

Characteristics of low IQ arsonists at psychiatric assessment. Med Sci Law, Vol. 48

No. 3, pp. 217-220.

Ducat, L., McEwan, T. and Ogloff, J.P. (2013). Comparing the characteristics of firesetting and non-firesetting offenders: are firesetters a special case? The Journal of Forensic Psychiatry \& Psychology, Vol. 24, pp. 549-569.

Enayati, J., Grann, M., Lubbe, S. and Fazel, S. (2008). Psychiatric morbidity in arsonists referred for forensic assessment in Sweden. The Journal of Forensic Psychiatry \& Psychology, Vol. 19 No. 2, pp. 139-147.

Fineman, K.R. (1995). A model for the qualitative analysis of child and adult deviant behavior. American Journal of Forensic Psychology, Vol. 13, pp. 31-60.

Hall, I., Clayton, P. and Johnson, P. 2005. Arson and learning disability. In: T.

Riding., C. Swann. \& Swann., B. (eds.) The Handbook of Forensic Learning

Disabilities. Oxford: Radcliffe Publishing.

Harris, G.T. and Rice, M.E. (1996). A typology of mentally disordered fire-setters. Journal of Interpersonal Violence, Vol. 22, pp. 351-363. 
Hogue, T., Mooney, P., Morrissey, C., Steptoe, L., Johnston, S., Lindsay, W.R. and Taylor, J.L. (2007). Emotional and behavioural problems in offenders with intellectual disability: comparative data from three forensic services. Journal of Intellectual Disability Research, Vol. 51 No. 10, pp. 778-785.

Hogue, T., Steptoe, L., Taylor, J.L., Lindsay, W.R., Mooney, P., Pinkey, L., Johnston, S., Smith, A.H.W. and O'Brien, G. (2006). A comparison of offenders with intellectual disability across three levels of security. Criminal Behaviour and Mental Health, Vol. 16, pp. 13-28.

Inciardi, J.A. (1970). The Adult Firesetter. Criminology, Vol. 8 No. 2, pp. 145-155. Johnston, S. (2004). Forensic Issues in learning disability. Criminal Behaviour and Mental Health, Vol. 14, pp. S53-S55.

Koson, D.F. and Dvoskin, J. (1982). Arson: A diagnostic survey. Bulletin of the American Academy of Psychiatry \& the Law, Vol. 10 No. 1, pp. 39-49.

Lees-Warley, G. and Rose, J. (2015). What does the evidence tell us about adults with low intellectual functioning who deliberately set fires? A systematic review. International Journal of Developmental Disabilities, Vol. 61 No. 4, pp. 242-256. Leong, G.B. and Silva, J.A. (1999). Revisiting arson from an outpatient forensic perspective. Journal of Forensic Sciences, Vol. 44, pp. 558-563.

Lindsay, W.R. (2002). Research and literature on sex offenders with intellectual and developmental disabilities. Journal of Intellectual Disability Research, Vol. 46 No. Supplement 1, pp. 74-85.

Lindsay, W.R., Hogue, T., Taylor, J.L., Steptoe, L., Mooney, P., O'Brien, G., Johnston, S. and Smith, A.H.W. (2008). Risk Assessment in Offenders With Intellectual Disability: A Comparison Across Three Levels of Security. International Journal of Offender Therapy and Comparative Criminology, Vol. 52 No. 1, pp. 90111. 
Long, C.G., Banyard, E., Fulton, B. and Hollin, C.R. (2014). Developing an assessment of fire-setting to guide treatment in secure settings: the St Andrew's Fire and Arson Risk Instrument (SAFARI). Behavioural and Cognitive Psychology, Vol. 42 No. 5, pp. 617-628.

Murphy, G.H. and Clare, I.C.H. (1996). Analysis of motivation in people with mild learning disabilities (mental handicap) who set fires. Psychology, Crime \& Law, Vol. 2, pp. 153-164.

O'Brien, G., Taylor, J.L., Lindsay, W.R., Holland, A.J., Carson, D., Steptoe, L., Price, K., Middleton, C. and Wheeler, J. (2010). A multi-centre study of adults with learning disbailities referred to services for antisocial or offending behaviour: demographic, individual, offending and service characteristics. Journal of Learning Disabilities and Offending Behaviour, Vol. 1 No. 2, pp. 5-15.

Quinsey, V.L., Book, A. and Skilling, T.A. (2004). A follow-up of deinstitutionalised developmentally handicapped men with histories of antisocial behaviour Journal of Applied Research in Intellectual Disabilities, Vol. 17, pp. 243-254.

Repo, E. and Virkkunen, M. (1997). Outcomes in a sample of Finnish fire-setters. Journal of Forensic Psychiatry and Psychology, Vol. 8 No. 1, pp. 127-137.

Rice, M.E. and Harris, G.T. (1991). Firesetters admitted to a maximum security psychiatric institution: Offenders and offenses. Journal of Interpersonal Violence, Vol. 11, pp. 364-375.

Ritchie, E.C. and Huff, T.G. (1999). Psychiatric aspects of arsonists. Journal of Forensic Science, Vol. 44, pp. 733-740.

Taylor, J.L., Novaco, R.W., Gilmer, B. and Thorne, I. (2002). Cognitive-behavioural treatment of anger intensity among offenders with intellectual disabilities. Criminal Behaviour and Mental Health, Vol. 12, pp. 282-293. 
Taylor, J.L., Thorne, I., Robertson, A. and Avery, G. (2002). Evaluation of a group intervention for convicted arsonsists with mild and borderline intellectual disabilities. Criminal Behaviour and Mental Health, Vol. 12 No. 4, pp. 282-293.

Wechsler, D. (1981). Manual for the Wechsler Adult Intelligence Scale-Revised (WAIS-R), The Psychological Corporation, New York.

World Health Organisation. (2010). "International Statistical Classification of Disease and Related Health Problems", avaliable at: http://apps.who.int/classifications/icd10/browse/2010/en -/R41.8 (accessed 11 July 2015). 
Table 1

Demographic Variables

\begin{tabular}{lccr}
\hline & Index Arson & Index Violent & Index Sex \\
& Offence & Offence & Offence \\
& $\mathbf{N = 1 8}$ & $\mathbf{N}=\mathbf{5 3}$ & $\mathbf{N}=\mathbf{6 3}$ \\
\hline Mean age at admission & $29.66(9.88 ; 17-48)$ & $29.80(9.00 ; 14-50)$ & $31.42(11.69 ; 16-$ \\
(SD; Range) & & & $59)$ \\
Mean age at time of & $49.22(14.04 ; 30-77)$ & $47.56(10.38 ; 30-79)$ & $48.65(11.89 ; 28-$ \\
assessment (SD; Range) & & & $73)$ \\
Mean prior convictions & $2.17(2.59 ; 0-8)$ & $3.81(7.13 ; 0-39)$ & $1.59(2.43 ; 0-10)$ \\
before 18 (SD; Range) & & & \\
Mean prior convictions & $5.17(10.48 ; 0-45)$ & $7.54(10.44 ; 0-43)$ & $3.79(5.63 ; 0-28)$ \\
after 18 (SD; Range) & & & \\
\hline
\end{tabular}


Table 2

Mean frequencies of fire setting and violent offending history, as well as comorbid mental disorder

\begin{tabular}{|c|c|c|c|c|c|}
\hline$(2)$ & $\begin{array}{l}\text { Index Arson } \\
\text { Offence } \\
\mathrm{N}=18\end{array}$ & $\begin{array}{c}\text { Index } \\
\text { Violent } \\
\text { Offence } \\
\mathbf{N}=\mathbf{5 3}\end{array}$ & $\begin{array}{l}\text { Index Sex } \\
\text { Offence } \\
\text { N=63 }\end{array}$ & $\chi^{2}$ & $V$ \\
\hline $\begin{array}{l}\mathrm{N} \text { with history of fire } \\
\text { setting }\end{array}$ & $8(44.4 \%)$ & $5(9.4 \%)$ & $7(11.1 \%)$ & $15.71 * * *$ & .34 \\
\hline $\begin{array}{l}\mathrm{N} \text { with violent offending } \\
\text { history }\end{array}$ & $6(33.3 \%)$ & $32(60.4 \%)$ & $18(28.6 \%)$ & $12.58 * *$ & .31 \\
\hline $\begin{array}{l}\mathrm{N} \text { with sexual offending } \\
\text { history }\end{array}$ & $3(16.6 \%)$ & $12(22.6 \%)$ & $33(52.4 \%)$ & $13.91 * * *$ & .32 \\
\hline $\begin{array}{l}\mathrm{N} \text { with comorbid mental } \\
\text { disorder }\end{array}$ & $12(66.6 \%)$ & $39(73.6 \%)$ & $29(46.0 \%)$ & $9.50 * *$ & .27 \\
\hline $\begin{array}{l}\mathrm{N} \text { of those with comorbid } \\
\text { mental disorder with } \\
\text { schizophrenia }\end{array}$ & $1(5.5 \%)$ & $16(30.2 \%)$ & $6(9.5 \%)$ & $10.62 * *$ & .28 \\
\hline $\begin{array}{l}\mathrm{N} \text { of those with comorbid } \\
\text { mental disorder with } \\
\text { personality disorder }\end{array}$ & $8(44.4 \%)$ & $24(55.3 \%)$ & $10(15.9 \%)$ & $13.23 * *$ & .31 \\
\hline
\end{tabular}

Note: $\mathrm{p}<0.05^{*}, \mathrm{p}<0.01^{* *}, \mathrm{p}<0.001^{* * *}$. 
Table 3

Mean (SD) SDRS clinician ratings split by arson, violent, and sex offences

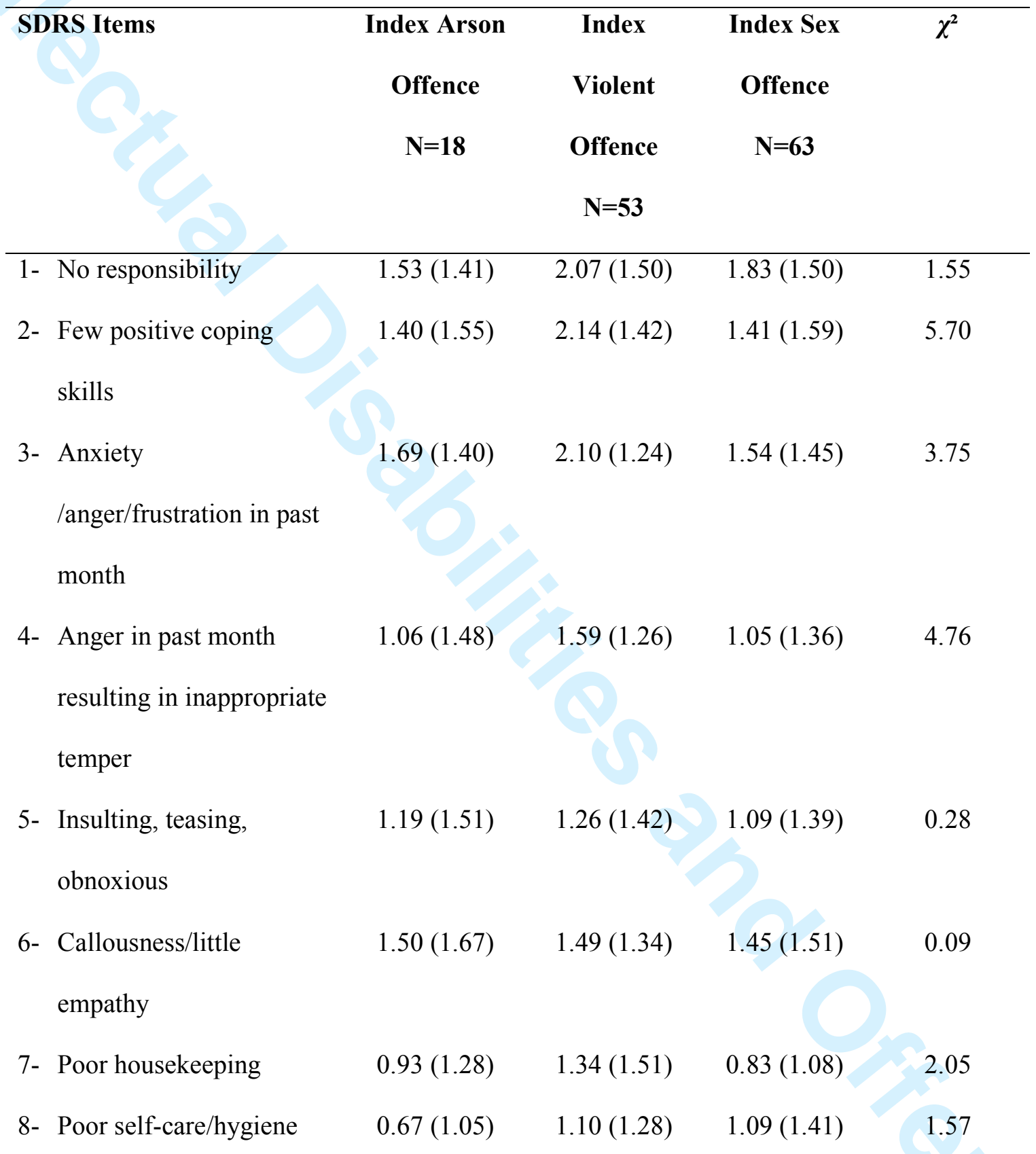

Note: $\mathrm{p}<0.05^{*}, \mathrm{p}<0.01^{* *}, \mathrm{p}<0.001^{* * *}$. Rating scale: $0=$ no problem, $2=$ moderate problem, 4=severe problem 